\title{
A New Synchrotron Approach to Study Ancient Materials: UV/Visible Photoluminescence Micro-Imaging
}

\author{
M Thoury ${ }^{1,2,}$ M Réfrégiers ${ }^{2}$ and L Bertrand ${ }^{2,1}$ \\ 1. SOLEIL synchrotron, Gif-sur-Yvette, France \\ 2. IPANEMA, USR 3461 CNRS, MCC, Gif-sur-Yvette, France
}

Photoluminescence has been exploited to characterize materials non-destructively since the early development of scientific studies on archaeological and cultural heritage materials [1]. First used as tool for qualitative visual examination of objects, luminescence spectroscopy, carried out at macro- and micro-scale, allowed characterizing luminescence properties of cultural heritage and artists' materials, such as organic binders or inorganic colorants [2]. More recently, multi-spectral imaging spectroscopy techniques have allowed to collect more quantitative information by combining the recording of spectroscopic data for each pixels of the image collected [3].

Photoluminescence properties can provide not only specific information on intrinsic characteristics of materials such as to identify the nature of crystalline defects in semiconductors or of metal ligand in organo-metallic complexes, but it can also be used as an efficient probe of materials' local environment. We defined new methodological protocols using DUV synchrotron beam to characterize minute quantities of organic and inorganic compounds encountered in art and archaeology, exploiting the full continuous tunability in energy and the brightness of the synchrotron source $[4,5]$.

Optimization of the instrumental set up allows a synergistic use of the two microscopes, allowing rasterscanning micro-spectrometry and full-field imaging, provided unprecedented coupling of sub-micrometric spatial resolution and 4 orders of magnitude in spatial dynamics, with concomitant spectral and spatial characterization of samples. In a recent study, we showed that historical zinc white powders that appear homogeneous at the macro-scale are highly heterogeneous at the micro- and nano-scales, and are markedly distinct from one another in that the relative intensities of the band-edge and the two defect-associated emissions observed at the sub-micrometer scale are very different [6].

Having established that we can identify and measure the diverse PL behaviour of different batches of $\mathrm{ZnO}$, we are interested in how it compares to particle shapes and size, and how it corresponds to known variation in chemistry, demonstrated by the variability in long-term stability of different paints. Based on this first study, promising development of this technique can lead it to find a more important place in the analytical sequences used to study art, palaeontology and archaeology materials.

\section{References:}

[1] JJ Rorimer, Metropolitan Museum of Art, New York, (1931)

[2] ER de la Rie, Studies in Conservation 27 (1982), 1-7, 65-69, 102-108.

[3] JK Delaney et al, Applied Spectroscopy 64, (2010), 584-594.

[4] M Thoury et al, Analytical Chemistry, 83 (2011), 1737-1745;

[5] L. Bertrand et al. Physics Reports 519 (2012), 51-96

[6] L Bertrand et al, Analyst, 138 (2013), 4463-4469 

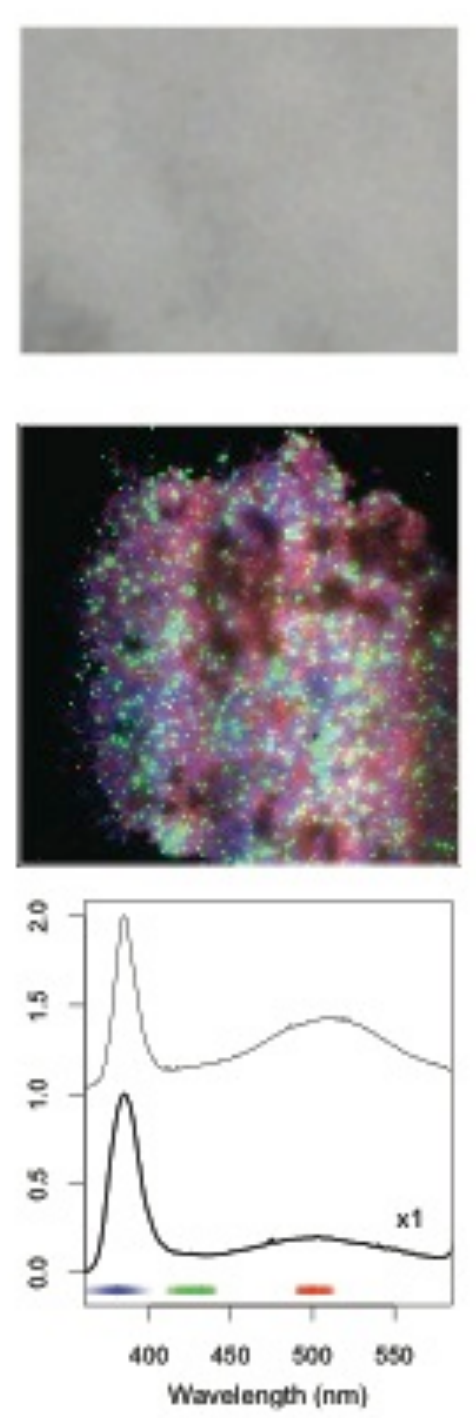
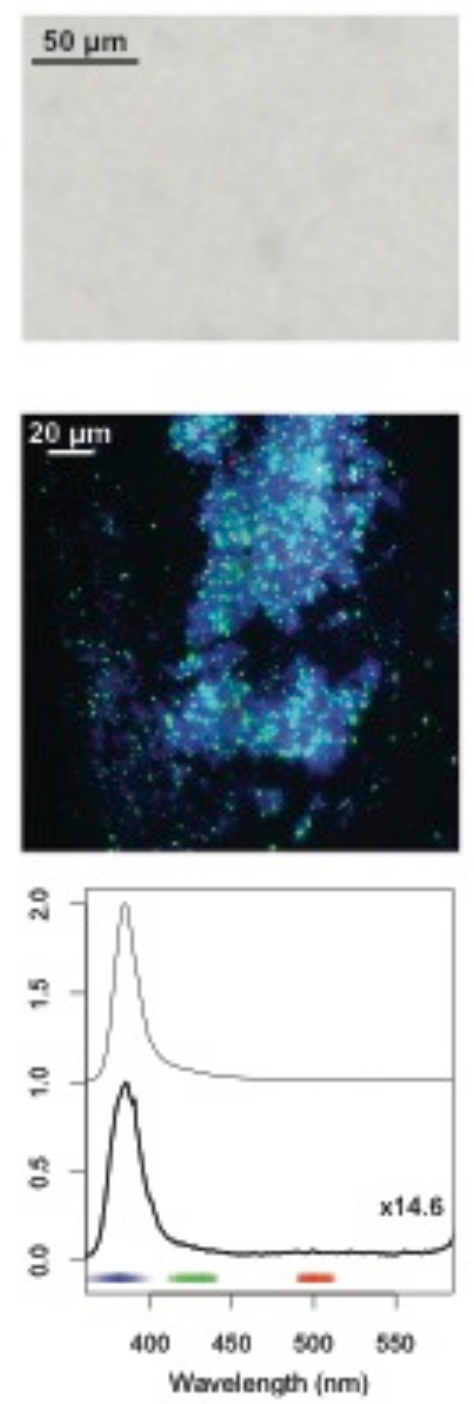
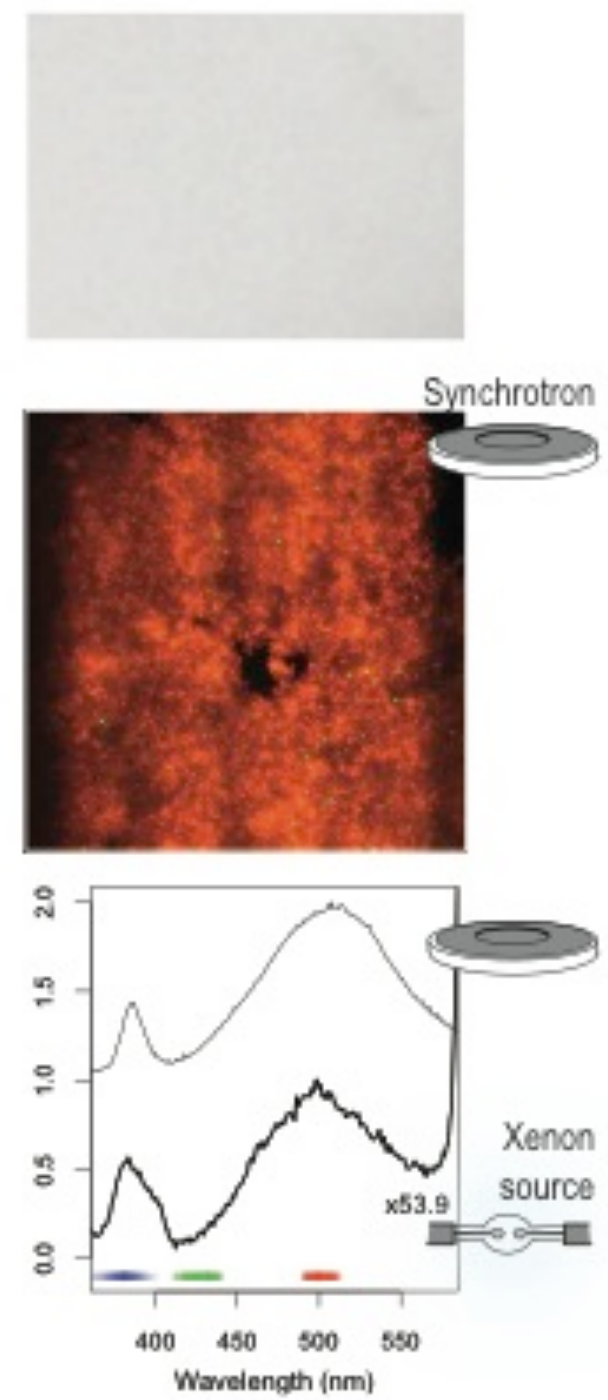

Figure 1. Study of three distinct historical zinc white pigments. From top to bottom: light microscopy images, synchrotron full-field UV PL images on a common intensity scale ( $40 \times$ objective), spectra from raster-scan synchrotron PL measurements (thin lines) and macroscopic PL spectra collected using the spectrofluorometer (thick lines). Intercomparison of PL full-field images is made possible by their rendering with identical intensity scales on each of the $R, G$ and $B$ bands. 